\title{
Nutrient Recovery Treatment Train Configuration and Optimization Using a New Model Library and Global Sensitivity Analysis
}

\section{Céline Vaneeckhaute, Filip H.A. Claeys, Erik Meers, Filip M.G. Tack, Evangelia Belia, Peter A. Vanrolleghem}

In the transition from waste(water) treatment plants (WWTPs) to waste(water) resource recovery facilities (WRRFs), the construction of accurate mathematical models requiring minimal computational effort to solve, is becoming an important challenge to hasten process and treatment train implementation and optimization. The present research aimed at the development of a generic nutrient recovery model (NRM) library based on detailed chemical solution speciation and reaction kinetics, with focus on fertilizer quality and quantity as model outputs. The purpose was to provide a common base that compiles the large body of knowledge on nutrient recovery processes currently available from research studies and operational experience. To this end, dynamic physicochemical three-phase process models for precipitation/crystallization (NRM-Prec), stripping (NRM-Strip), and acidic air scrubbing (NRM-Scrub) as key units were developed. In addition, a compatible biological-physicochemical anaerobic digester model (NRM-AD) was constructed. The latter includes sulfurgenesis, biological nitrogen $(\mathrm{N})$, phosphorus $(\mathrm{P})$, potassium $(\mathrm{K})$, and sulfur $(\mathrm{S})$ release/uptake, interactions with organics, among all other relevant processes, such as precipitation, ion pairing, and liquid-gas transfer.

Each dynamic mathematical model was built using: 1) the definition of a chemical speciation model using geochemical modelling software (PHREEQC/MINTEQ), 2) the description of a physicochemical and biochemical transformation model tailored to the models developed in the first step, and 3) the selection of a reactor mass balance model to describe the (time-dependent) process conditions. In order to facilitate numerical solution, an efficient procedure for calling PHREEQC (to obtain selected chemical speciation outputs) from the Modelica-coded kinetic transformation models using the Tornado/WEST software kernel was developed. A reduction of execution time was established at two critical points during model simulations: 1) the uploading and reading of the database and input files (through PHREEQC model reduction), and 2) the transfer of data between PHREEQC and Tornado (through tight model coupling). A three-to-five fold improvement of model simulation speed was obtained using the developed reduced models as compared to full PHREEQC and MINTEQ databases, respectively. An important discovery concerns the omission of essential physicochemical components, species, and reactions in view of nutrient recovery, for example, aluminum phosphate, potassium struvite, and ammonium sulfate precipitation, in the existing standard geochemical databases. Because of these flaws, a generic database with the purpose of nutrient recovery was created for future applications.

After implementation, the models were subjected to a battery of tests to ensure implementation correctness, also referred to as model verification. As such, typing errors, inconsistencies, gaps, and conceptual errors were eliminated, while bugs in the required software extensions were discovered and dealt with. Next to verification, also model validation was performed by comparison of the model outcomes with independent experimental data. Validation is often neglected in mathematical modelling, but is a key component of Good Modelling Practice. Simulation results using default parameters 
showed good agreement with experimental results under steady state conditions. Moreover, the ability of the models to act as a tool for increased process understanding and optimization was successfully demonstrated. Detailed chemical input characterization and chemical solution speciation were found to be of prime importance for modelling of nutrient recovery systems. Further research in terms of determination of precipitation/dissolution and gas transfer kinetics in real waste matrices is required to accurately calibrate and validate the models under dynamic conditions.

Global sensitivity analysis (GSA) was performed to select the most important factors influencing the model outputs among: 1) input waste characteristics, 2) process operational factors, and 3) kinetic parameters incorporated in the NRM's. Next to the relevance of the outcomes in terms of model validation, the GSA results allowed for listing generic recommendations for factor prioritiziation in view of future research. For all models, the variation in input waste composition resulted in major output variation through its direct effect on the operational $\mathrm{pH}$ and ionic strength. This underlines the fundamental importance of the accurate chemical solution speciation provided by the NRM's. Main impacts were found to be generalizable for different waste sources, showing the wide relevance of the study. Moreover, valuable insights in the interactions between unit process inputs and outputs were obtained through GSA. Major findings include, among others, the impact of chloride $(\mathrm{Cl})$ inhibition on ammonia removal in the stripping unit, the impact of calcium $(\mathrm{Ca})$, iron $(\mathrm{Fe})$, and aluminium $(\mathrm{Al})$ inhibition on $\mathrm{P}$ recovery in the precipitation unit, and the interaction between $\mathrm{Fe} / \mathrm{Al}, \mathrm{S}$, and methane $\left(\mathrm{CH}_{4}\right)$ production in the anaerobic digester.

Based on the results, it was possible to set up an optimal treatment train configuration for nutrient recovery that maximizes resource recovery at minimal cost (Fig. 1).

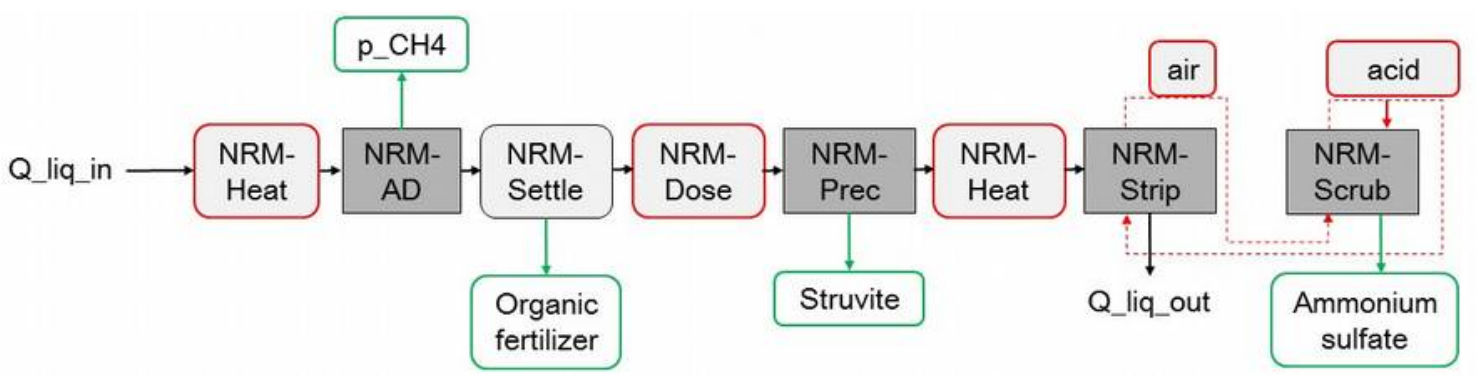

Figure 1: Proposed treatment train configuration targeting struvite and ammonium sulfate fertilizer. Red $=$ consumable $(=$ cost $)$. Green $=$ recovered resource $(=$ revenue $)$. $\mathrm{AD}=$ anaerobic digestion; Dose $=$ chemical dosing; Heat $=$ heat exchanger; Prec $=$ precipitation/crystallization; $\mathrm{p}=$ partial pressure in the biogas; $\mathrm{Q}_{-}$liq $=$liquid flow rate; Scrub $=$ scrubber; Strip $=$ stripper.

Finally, the use of the NRM library to establish the operational settings of a sustainable and cost-effective treatment scenario with maximal resource recovery and minimal 
energy and chemical requirements was demonstrated for pig manure as a case study. Under the optimized conditions and assumptions made, potential financial benefits for a large-scale anaerobic digestion and nutrient recovery project were estimated at 2.8-6.5 USD $\mathrm{m}^{-3}$ manure based on net variable cost calculations, or an average of $\pm 2 \mathrm{USD} \mathrm{m}^{-3} \mathrm{y}^{-}$ ${ }^{1}$, equivalent with $40 \mathrm{USD}^{-1}$ total solids $\mathrm{y}^{-1}$, over 20 years when also taking into account capital costs. Hence, it is likely that in practice a full-scale 'ZeroCostWRRF' can be constructed. Nevertheless, subsidies and heat balances were found to play a crucial role in determining the feasibility of resource recovery projects. Moreover, the optimal treatment train configuration and operating conditions were found to be dependent on local legislations and fertilizer markets.

It can be concluded that the NRM library and GSA strategy developed in this study provide a valuable and cost-effective framework for increased process understanding, treatment train configuration, and optimization of region-specific nutrient recovery applications. Starting from the obtained results and insights, a generic roadmap for setting up nutrient recovery strategies as function of fertilizer markets and input characteristics was proposed. The roadmap involves: 1) an overview of bio-based fertilization recommendations as function of fertilizer legislations, 2) guidelines for determining the feasibility of nutrient recovery based on operational experience, and 3) an algorithm for configuration and optimization of nutrient recovery treatment trains as function of input waste characterization and fertilizer markets. As such, the roadmap provides useful guidance for waste(water) processing utilities considering the implementation of nutrient recovery practices. This, in turn, should stimulate and hasten the global transition from traditional WWTPs to WRRFs. 